\title{
Heart rate variability analysis
}

\author{
L. Hejjel, ${ }^{1}$ I. Gál ${ }^{2}$ \\ ${ }^{1}$ Department of Cardiac Surgery, Heart Institute, Medical Faculty, University of Pécs, Pécs \\ ${ }^{2}$ Teaching Department of Surgery, Bugát Pál Hospital, Gyöngyös, Hungary
}

Received: January 12, 2002

Accepted: February 6, 2002

\begin{abstract}
The expansion of heart rate variability analysis has been facilitated by the remarkable development of computer sciences and digital signal processing during the last thirty years. The beat-to-beat fluctuation of the heart rate originates from the momentary summing of sympathetic and parasympathetic influences on the sinus node. According to the extensive associations of the autonomic nervous system, several factors affect heart rate and its variability such as posture, respiration frequency, age, gender, physical or mental load, pain, numerous disease conditions, and different drugs. Heart rate variability can be quantitatively measured by time domain and frequency domain methods that are detailed in the paper. Non-linear methods have not spread in the clinical practice yet. Various cardiovascular and other pathologies as well as different forms of mental and physical load are associated with altered heart rate variability offering the possibility of predicting disease outcome and assessing stress.
\end{abstract}

Keywords: heart rate variability, stress, sinus node, sympathetic and parasympathetic influences, autonomic nervous system

Beat-to-beat blood pressure level and heart rate fluctuations related to respiratory cycle were recognized as early as in the 18 th century by Hales. However, the first real clinical application of heart rate variability (HRV) analysis has been brought up at the beginning of electrical fetal monitoring: Hon and Lee published in 1963 that certain changes in the RR-interval pattern precede the heart rate changes occurring at fetal distress. Ten years later Sayers used power spectral estimation for HRV assessment and identified low-frequency components in heart rate alterations besides the high-frequency respiratory changes. The elevated mortality of postinfarction patients with reduced HRV was shown first by Wolf et al. in 1977. Akselrod and his co-workers demonstrated the

Correspondence should be addressed to

Laszló Hejjel, MD

Medical Faculty, University of Pécs

Heart Institute, Department of Cardiac Surgery

H-7624 Pécs, Ifjúság út 13, P.O. Box 99, Hungary

Phone/Fax: +36 72 324-358, +36 72 324-122, +36 72 326-222 
vegetative nervous system as a moderator of HRV changes in $1981(35,45)$. Following these initial discoveries the number of researchers and publications investigating HRV have exponentially increased owing to the improvements and spreading of computerized ECG systems. Since then comprehensive observations have been made in several pathologies, drug effects, maneuvers, physical and mental stress situations.

\section{Physiological background of heart rate fluctuations}

In physiologic circumstances the sinus node controls the actual heart rate as a primary pacemaker. It has both sympathetic and parasympathetic innervations, and several autocrine, paracrine and endocrine substances effect on it. The sinus node acts as the final summing element of sympathetic and parasympathetic stimuli and their instantaneous relation is reflected in the actual interbeat interval. The action of these factors shows different time-courses which produces certain spectral bands in the beatto-beat fluctuation (32).

Parasympathetic efferent nerve fibers come through the parasympathetic ganglia positioned in the periaortic and epicardial fat pad. Efferent sympathetic innervation arrives from superior, middle, and inferior cervical and the upper four or five thoracic ganglia. Medullary nuclei and reticular formation give both excitatory and inhibitory preganglionic efferent fibers as well as accept afferent fibers. Afferent pathways from baroreceptors and so-called cardiopulmonary receptors to the brain stem are closing the loop assuring a feedback mechanism. These baroreceptors are located in the wall of aortic arch and great arteries arising from it, and in the carotid sinus. The hypothalamus is considered the most important supramedullary compound that integrates autonomic, somatic, mental and emotional information via its extensive associations (Fig. 1) $(1,32,46)$.

\section{Methods for estimating HRV}

Investigating neurocardiac modulation, the attention is focused on the discharge frequency of the sinus node. However, because of practical considerations a more exactly definable point of the ventricular complex is preferred as a reference point in spite of some alteration picked up in the conduction system from the sinus node to the ventricular myocardium. The time intervals between the consecutive R-peaks or between the mid-height points of the ascending slope of consecutive ventricular complexes are determined on the digitized, good quality electrocardiogram. The series of these values (usually in milliseconds) plotted against their serial number gives the tachogram as the root of all further calculations (Fig. 2). 


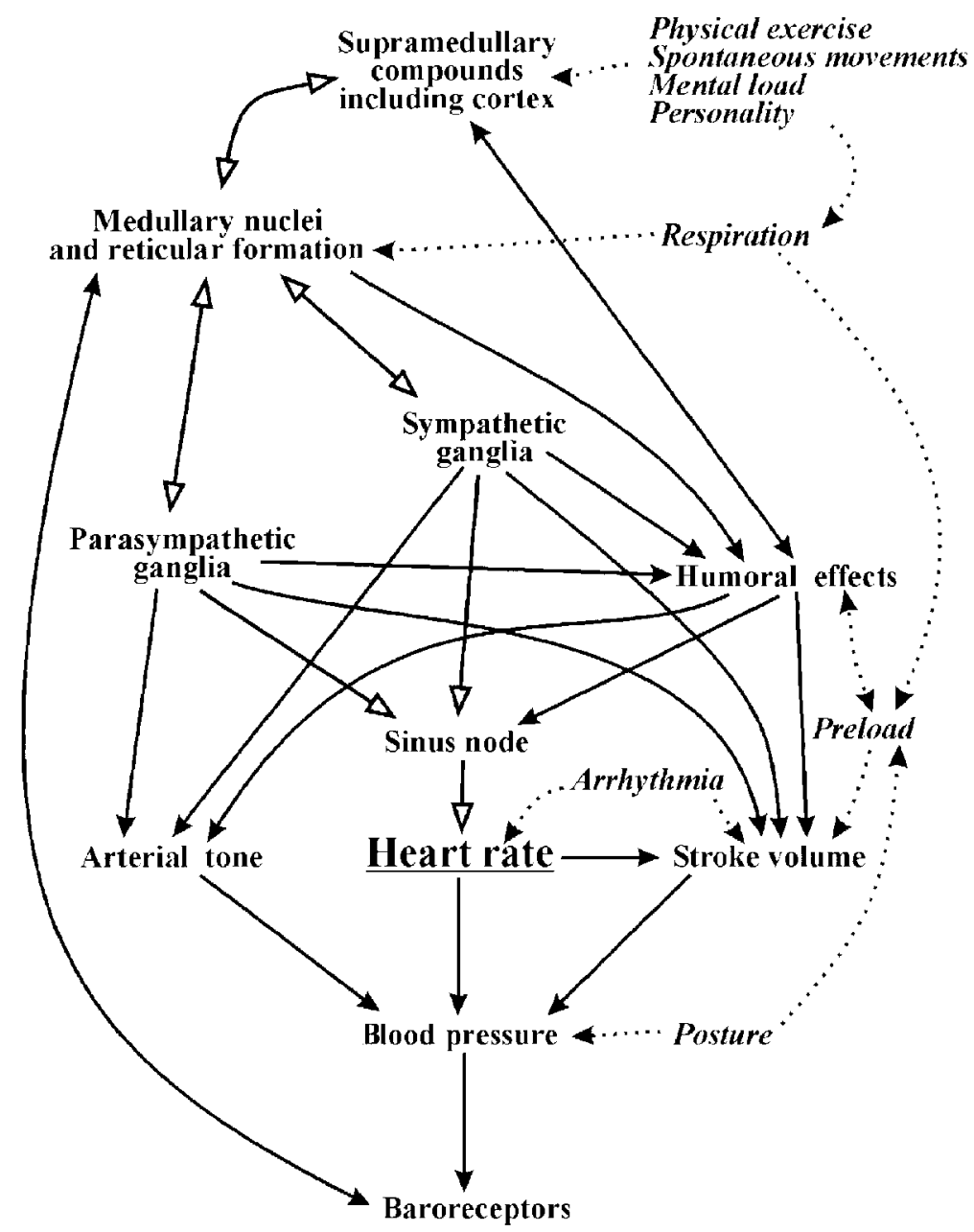

Fig. 1. Physiological control of heart rate and heart rate variability in brief. Some of the most important factors influencing heart rate are cited in Italic

The tachogram is expected to contain exclusively normal sinus interbeat intervals in order to get accurate parameters of HRV. Every other kind of beats or artifacts must be rejected or corrected. The ratio of erroneous beats depends on the robustness of the applied R-detector algorithm (missing or spurious beats), and the cardiovascular status of the observed individual (ectopic beats, blocked sinus revolution). 


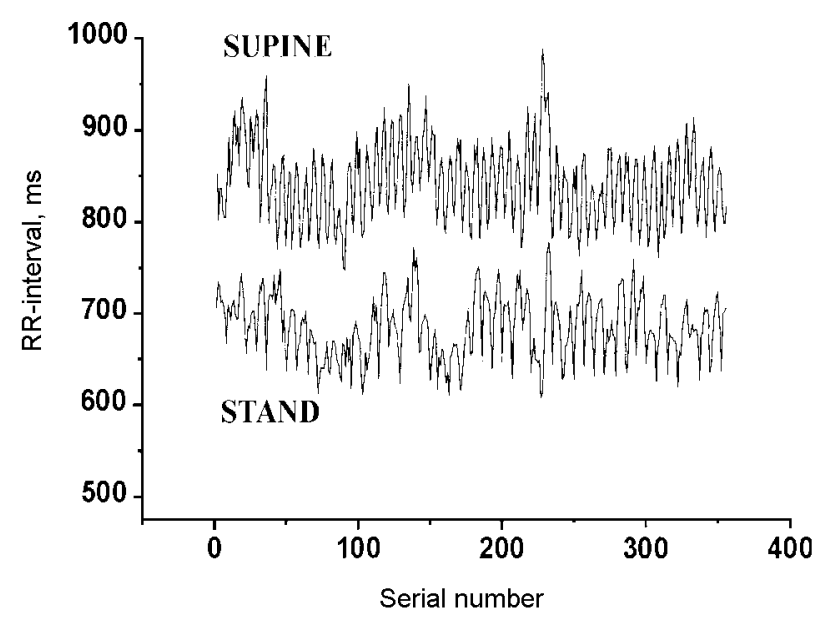

Fig. 2. Tachograms of the same person during standing and supine position. The actual RR-interval duration plotted against the serial number gives a tachogram. Elevated heart rate and a smoother waveform indicating reduced variability can be seen in standing, as shown by the time domain parameters. Supine: $n=355$, mean=843.61 ms, SDNN=42.68 ms, RMSSD=36.50, PNN50=17.18\%. Standing: $\mathrm{n}=355$, mean=683.43 ms, $\mathrm{SDNN}=33.16 \mathrm{~ms}, \mathrm{RMSSD}=26.36 \mathrm{~ms}, \mathrm{PNN} 50=7.06 \%$. (SDNN-standard deviation of normal-to-normal RR-interval duration, RMSSD-root mean square of successive RR-differences, PNN50-percentage of interbeat RR-differences greater than $50 \mathrm{~ms}$ )

During sinus rhythm there are no sudden changes in the heart rate, so the simplest filtering method can be achieved by eliminating the actual RR-interval value (or even the next one too) if it exceeds the $\pm 20 \%$ of the recently accepted interval. Usually additional manual checking is recommended. One method is just removing a rejected beat or it may be replaced by the linear or exponential interpolation of the neighboring normal-to-normal (NN-) intervals $(32,45)$.

The simplest and oldest time domain methods are the mean heart rate or cycle length, the difference or the ratio of the shortest and longest $\mathrm{NN}$-interval on the recorded ECG. Computerized data storage and signal processing allowed the use of more sophisticated approaches based on statistical calculations like standard deviation (SDNN), standard deviation of 5-minute average of NN-intervals (SDANN), root mean square of successive NN-differences (RMSSD) and the percentage of NN-differences greater than $50 \mathrm{~ms}$ (PNN50). While the standard deviation gives the average divergence around the mean, the RMSSD shows the mean divergence between adjacent NN-intervals. Consequently, the RMSSD as well as PNN50 represent beat-to-beat (short-time) changes more accurately than SDNN. The value of time domain parameters strongly depends on the length of the analyzed time interval, therefore five-minute and 24-hour long recordings are recommended for short and long-term measurements as standards (45). Geometric methods based on the histogram of the NN-intervals are 
considered in the time domain as well. The bin size of the histogram is usually $1 / 128$ second $(7.8125 \mathrm{~ms})$ according to the sampling rate of conventional Holter monitors. The modal duration is the most frequently observed $\mathrm{NN}$-interval. Modeling the histogram by a triangle, its baseline width can be equal to the quotient of its doubled area and its height. The height equals to the count in the modal bin and the area can be estimated by the total amount of $\mathrm{NN}$-intervals. Thus, the triangular index (TI) can be obtained by the quotient of the total and modal number of NN-intervals. The triangular interpolation (TINN) equals the baseline-width of a triangle given by its apex as the top of the modal bin and the two sides approximating the two slopes of the histogram by linear regression. The top angle index (TAI) represents the angle in the opposite of the baseline of the fitted triangle in grades (Fig. 3). These histogram-based parameters highly correlate with statistical methods $(33,50)$.

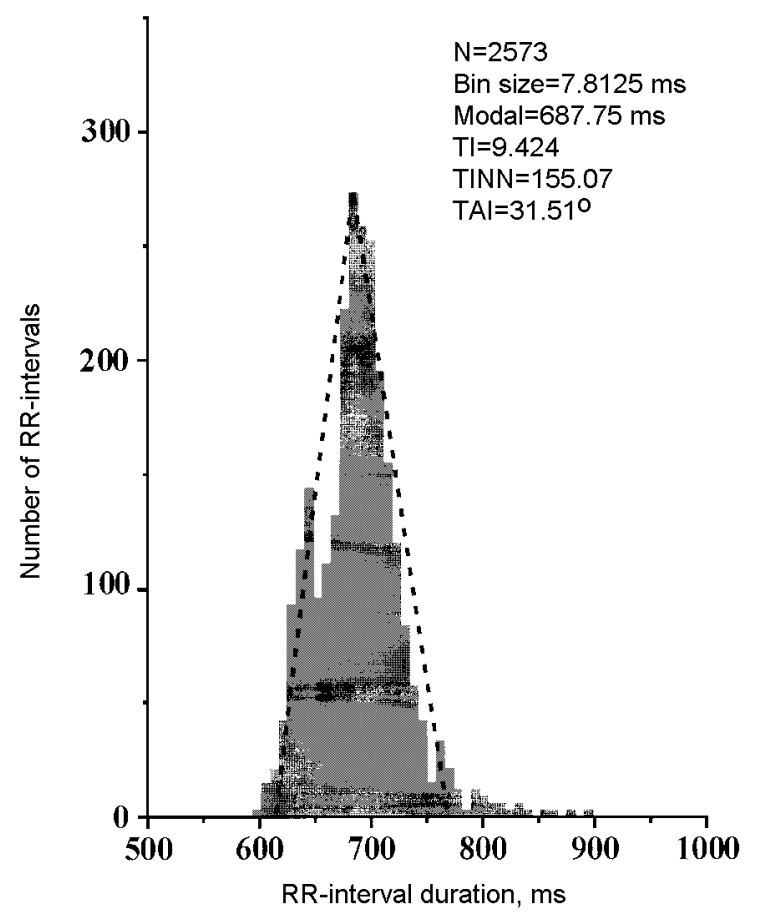

Fig. 3. A histogram of 2573 RR-interval durations. The modal value is the number of the most frequently observed RR-interval. Modeling the histogram by a triangle, relatively noise-resistant quantitative parameters can be achieved. Triangular index (TI) is the quotient of the area of the triangle and its height, and can be simply computed by the ratio of the number of all RR-intervals and the amount of RR-intervals in the modal bin. Triangular interpolation (TINN) represents the length of the base of the triangle fitted by linear regression. Top angle index equals the angle opposing the baseline of this triangle in grades. Histogram-based methods are reliable on at least 20 minute long or longer ECG-records 


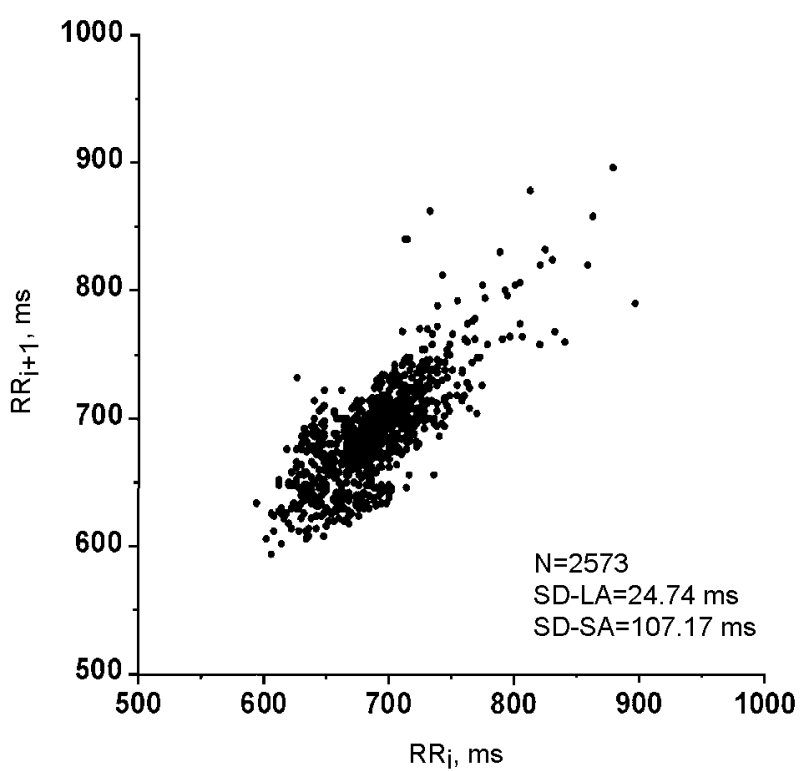

Fig. 4. Return map or Lorentz-plot of 2573 RR-interval durations. Correlation of a data series can be investigated by plotting the (i+1)-th element against the (i)-th element. The expansion and the shape of the set of points represents the irregularity of the original series. The directional standard deviation parallel to the unitary slope line passing through the origo represents long-term variability (long axis, SD-LA), whereas the standard deviation in the perpendicular direction corresponds to short-term changes (short axis, SD-SA).

The use of this method is suggested on long-time tachograms

Another graphic representation of heart period sequences can be the return map or Lorentz-plot (or Poincaré-plot), where the $\mathrm{NN}_{\mathrm{i}+1}$ is plotted against the $\mathrm{NN}_{\mathrm{i}}$ in Cartesian coordinates resulted in a map of dots (Fig. 4). Its shape and expansion can be assessed visually. The lesser is the area the smaller is the variability, and vice versa. The long axis of the plot fits on the unitary slope line passing through the origin, whereas the short axis is perpendicular to that. They cross each other in the center point, which is located at the coordinates of the mean RR-duration. Quantitative analysis can be performed by the calculation of the standard deviation of data points in the direction of the long (SD-LA) or short (SD-SA) axis, the length of both axes equals to the related standard deviation. The first one represents the short-term, while the last one shows the long-term variability. Return map is originally a non-linear method, however, the abovementioned inherited parameters are insensitive to the non-linear characteristics, practically they are equivalent with the popular time domain methods as has recently been published (5). Geometric methods can be performed on at least 20-minute-long or (especially the Lorentz-plots) even longer tachograms. They are relatively robust against noise and non-sinus beats $(33,50)$. 


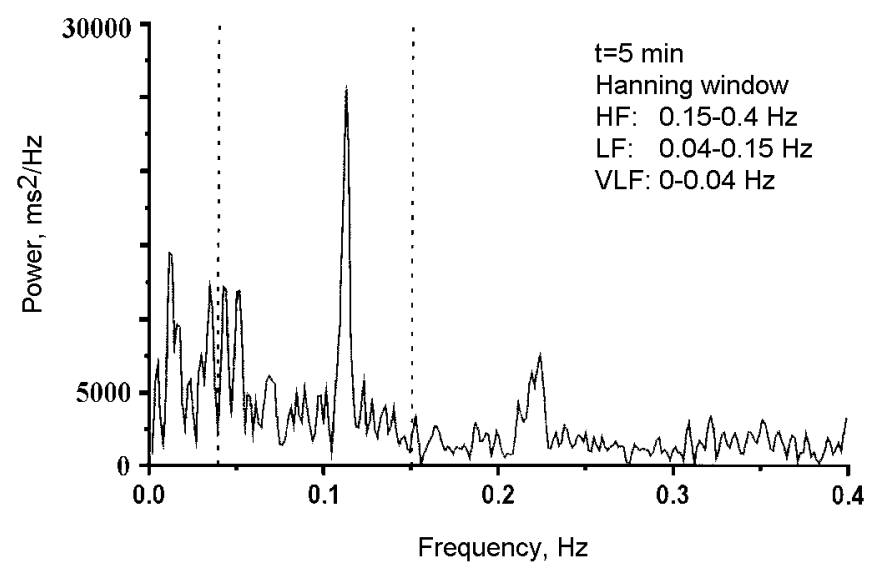

Fig. 5. Spectral analysis of a 5-minute-long tachogram by fast Fourier-transformation. Spectral bands are separated by dashed vertical lines. The peak in the high-frequency (HF) band reflects respiratory arrhythmia induced by about 14 breath/min. (very-low-frequency: VLF, low-frequency: LF band)

Frequency domain measures give a spectral analysis of the tachogram of 200-500 beats by showing the relative (normalized unit-NU) or absolute $\left(\mathrm{ms}^{2} / \mathrm{Hz}\right)$ contributions of certain frequencies to the total variance, or in simple terms: plotting the variance as a function of frequency (Fig. 5). Mathematically two distinct methods are available for this purpose: Fast Fourier Transformation (FFT) and Autoregressive Modeling. The more general FFT requires data series consisting of equidistant points as a function of time; therefore the irregularly time-sampled tachogram must be resampled at a fixed rate of 250-330 ms. In order to avoid the aliasing phenomenon, a consecutive low-pass digital filtering is suggested below the half value of the resampling frequency (Nyquist frequency) before performing FFT. Removing the 'DC-component' of the tachogram is possible by subtracting the mean RR-interval duration. Windowing of the resampled tachogram is recommended to prevent the generation of harmonic artifacts resulting from the finite-length tachograms. On the spectral distribution chart of short-term heart rate tachograms high- (HF: 0.4-0.15 Hz), low- (LF: 0.15-0.04 Hz) and very low- (VLF: $<0.04 \mathrm{~Hz}$ ) frequency bands are discerned. The HF variability represents the respirationcoupled arrhythmia and is considered as a result of purely parasympathetic influence, whereas the LF component is a consequence of both sympathetic and parasympathetic control. The ratio of the LF and HF power is a good indicator of sympathovagal balance. VLF power reflects long-term neuroendocrin fluctuations $(3,21,32,45,50)$.

Physiological parameters demonstrate neither regular nor random dynamics in time (and in space). They show a special behavior called nonlinear or chaotic, as a result of complex feedback mechanisms of biological systems. Statistical methods are appropriate for evaluating random data series; while spectral techniques are 
suitable for describing periodic signals. Using either of them for analyzing chaotic biological sequences, significant information on their dynamics may be lost. Just enumerating some non-linear parameters: fractal dimension, approximate entropy, Lyapunov exponents, and correlation dimension. However, nonlinear methods have not yet been spreading in the clinical practice because of their abstract and sophisticated approach and presentation, they are expected to be developed to a powerful tool for variability analysis in the near future $(10,14,15,49,50)$.

\section{Clinical relevance of $H R V$}

The publication of Kleiger (22) oriented the attention to the prognostic value of reduced HRV in cardiovascular disease. Most parts of the studies investigates the HRV in patients with an acute myocardial infarction. It has been proved to be an independent risk factor for mortality, postinfarction arrhythmias and disease progression and may be used for risk-stratification of patients as well $(3,8,27,33,42)$. Reduced HRV and its prognostic role were observed in congestive heart failure $(6,48)$, hypertension $(25)$, in diabetic patients $(13,50)$, and in multiple metabolic syndrome (29). Before the onset of ventricular tachyarrhythmias reduced HRV was observed that is suggestive of vagal withdrawal and sympathoexcitation $(19,39)$. Autonomic changes reflected in heart rate variability alterations also play an important role in ambulatory ischemic events (23). Aging decreases the variability estimated by traditional time and frequency domain as well as by non-linear methods $(30,38)$. According to a study performed on healthy volunteers the HRV is lower in women compared to men that may be elucidated by reduced sympathetic influence considering female autonomic nervous system cardioprotective (40). Exposure to particulate air pollution also alters cardiac vegetative control directly and via released inflammatory cytokines (31). The increasing number of studies in different pathologies and on experimental models can result in deeper insight into the principal mechanisms of HRV changes and its associations to the underlying disease (19).

\section{Effects of mental and physical stress on HRV}

Reduction of HRV has been published during memory search (41) and mental arithmetic $(18,24,44)$ reflecting vagal withdrawal at least in part. All frequency bands of HRV are dominantly under parasympathetic control during mental load (26). Reduction of mid-frequency $(0.07-0.13 \mathrm{~Hz})$ peak by mental arithmetic highly correlated in dizygotic but not monozygotic twins, suggesting considerable genetic involvement in 
the control of HRV (24). Verbalization during mental activity noticeably influences HRV through alterations in respiratory frequency $(2,44)$. Left monocular viewing causes a significant elevation of the mid-frequency $(0.07-0.13 \mathrm{~Hz})$ peak (47).

Physical exercise significantly decreases both low and high frequency power spectrum of HRV $(7,36)$, however, others found HRV increased during static exercise compared to dynamic exercise or rest (16). In another study moderate treadmill run significantly increased low frequency component of the power spectrum (43). Spontaneous movements also can affect HRV that must be taken into account (11). The beneficial effects of regular physical exercises in many cardiovascular disorders may be related to the increase in parasympathetic tone and consequent elevation of HRV observed both in young and older men following a six-month aerobic exercise training (28).

A new application for HRV analysis: assessing the mental load of the surgeon during different operations

Laparoscopic surgery versus open surgery has great advantages for the patient: small incisions, intact abdominal wall, less postoperative pain, shorter reconvalescence, and cosmetically better results. On the other hand, two-dimensional sight, special instrumentation, loss of palpation and direct contact with tissues make endoscopic operations much more demanding for the surgeon compared to open methods. Elevated heart rate of the surgeons during performing an operation has been recognized several years ago $(12,37)$ as well as their reduced HRV during every phase of the operation and immediately after surgery (9). According to our preliminary studies (17) HRV analysis in the time domain showed reduced variability during laparoscopic operation compared to open procedures. These data are consistent with those published by Böhm et al. (4), where significant reduction of the LF/HF (low and high frequency band) ratio was observed during laparoscopic versus conventional sigmoid resection. This was a reflection of augmented sympathetic control due to increased mental strain.

\section{Conclusions}

HRV can be measured exactly and reproducibly by time domain, frequency domain and non-linear methods. The extensive associations of the neural network regulating heart rate allow the investigation of the action of several internal and external factors. On the other hand, it makes the analysis difficult, because clear or "sterile" circumstances are needed to exclude unwanted influences. In the clinical practice, it is rarely feasible. Well-standardized $\mathrm{HRV}$-tests are needed respecting as much conditions 
as possible including breathing-rhythm, posture, slight movements, and mental activity among others. The results must be age- an sex-matched. Inter-individual differences can be filtered out by comparing two- or multiple-stage tests. However, persisting comorbidities may remain a problem in the aspect of an individual disease condition.

HRV analysis can be an effective method for comparing stress situations/reactions, labor hygiene and ergonomical measures beside prognosing survival and disease progression in different pathologies, however careful presentation and evaluation are necessary.

\section{REFERENCES}

1. Aubert AE, Ramaekers D: Neurocardiology: the benefits of irregularity. Acta Cardiol. 54(3), 107-120 (1999)

2. Bernardi L, Wdowczyk-Sculc J, Valenti C, Castoldi S, Passino C, Spadacini G, Sleight P: Effects of controlled breathing, mental activity and mental stress with or without verbalization on heart rate variability. J. Am. Coll. Cardiol. 35, 1462-1469 (2000)

3. Bigger JT, Fleiss JL, Rolnitzky LM, Steinman RC: The ability of several short-term measures of RR variability to predict mortality after myocardial infarction. Circulation 88, 927-934 (1993)

4. Böhm B, Rötting N, Schwenk W, Grebe S, Mansmann U: A prospective randomized trial on heart rate variability of the surgical team during laparoscopic and conventional sigmoid resection. Arch. Surg. 136, 305-310 (2001)

5. Brennan M, Palaniswami M, Kamen P: Do existing measures of Poincaré plot geometry reflect nonlinear features of heart rate variability? IEEE Trans. Biomed. Eng. 48, 1343-1347 (2001)

6. Casolo G, Balli E, Taddei T, Amuhasi J, Gori C: Decreased spontaneous heart rate variability on congestive heart failure. Am. J. Cardiol. 64, 1162-1167 (1989)

7. Cottin F, Papellier Y, Escourrou P: Effects of exercise load and breathing on heart rate and blood pressure variability during dynamic exercise. Int. J. Sports Med. 20, 232-238 (1999)

8. Cripps TR, Malik M, Farrell TG, Camm AJ: Prognostic value of reduced heart rate variability after myocardial infarction: clinical evaluation of a new analysis method. Br. Heart J. 65, 14-19 (1991)

9. Czyzewska E, Kiczka K, Czarnecki A, Pokinko P: The surgeon's mental load during decision making at various stages of operations. Eur. J. Appl. Physiol. Occup. Physiol. 51, 441-446 (1983)

10. Denton TA, Diamond GA, Helfant RH, Khan S, Karagueuzian H: Fascinating rhythm: a primer on chaos theory and its application to cardiology. Am. Heart. J. 120, 1419-1440 (1990)

11. Fortrat JO, Formet C, Frutoso J, Gharib C: Even slight movements disturb analysis of cardiovascular dynamics. Am. J. Physiol. 277(46), H261-H267 (1999)

12. Foster GE, Evans DF, Hardcastle JD: Heart-rates of surgeons during operations and other clinical activities and their modulation by oxprenolol. Lancet 1, 1323-1325 (1978)

13. Freeman R, Saul JP, Roberts MS, Berger MD, Broadridge C, Cohen RJ: Spectral analysis of heart rate in diabetic autonomic neuropathy. A comparison with standard tests of autonomic function. Arch. Neurol. 48, 185-190 (1991)

14. Glenny RW, Robertson HT, Yamashiro S, Bassingthwaighte JB: Applications of fractal analysis to physiology. J. Appl. Physiol. 70, 2351-2367 (1991)

15. Goldberger AL: Physionet. Nonlinear dynamics, fractals, and chaos theory: implications for neuroanatomic heart rate control in health and disease. http://www.physionet.org/tutorials/ndc (1999) 
16. Gonzalez-Camarena R, Carrasco-Sosa S, Roman-Ramos R, Gaitan-Gonzalez MJ, Medina-Banuelos V, Azbiroz-Leehan J: Effect of static and dynamic exercise on heart rate and blood pressure variabilities. Med. Sci. Sports Exerc. 32, 1719-1728 (2000)

17. Hejjel L, Gal I: Assessment of operative stress during laparoscopic surgery. Preliminary studies. SAGES Congress, Atlanta, GA, USA (2000)

18. Hejjel L: Stressz becslése a szívritmus variabilitásával. XVII. Magyar Kísérletes Sebészeti Kongresszus, Szeged, 1999. szept. 16-18.

19. Huikuri HV, Mäkikallio T, Airaksinen KEJ, Mitrani R, Castellanos A, Myerburg RJ: Measurement of heart rate variability: a clinical tool or a research toy? J. Am. Coll. Cardiol. 34, 1878-1883 (1999)

20. Huikuri HV, Seppänen T, Koistinen MJ, Airaksinen KEJ, Ikäheimo MJ, Castellanos A, Myerburg R: Abnormalities in beat-to-beat dynamics of heart rate before the spontaneous onset of life-threatening ventricular tachyarrhythmias in patients with prior myocardial infarction. Circulation 93, 1836-1844 (1996)

21. Kékes E (1998): Szívfrekvencia variabilitás mérése. In: EKG enciklopédia, Springer Orvosi Kiadó Kft., Budapest, pp. 280-285.

22. Kleiger RE, Miller JP, Bigger JT, Moss AJ and the Multicenter Post-infarction Research Group: Decreased heart rate variability and its association with increased mortality after acute myocardial infarction. Am. J. Cardiol. 59, 256-262 (1987)

23. Kop WJ, Verdino RJ, Gottdiener JS, O'Leary ST, Bairey MCN, Krantz DS: Changes in heart rate and heart rate variability before ambulatory ischemic events (1). J. Am. Coll. Cardiol. 38, 742-749 (2001)

24. Láng E, Szilágyi N, Metneki J, Weisz J: Effects of mental load on the spectral components of heart period variability in twins. Acta Biochim. Biophys. Hung. 26, 111-120 (1991-1992)

25. Langewitz W, Ruddel H, Schachinger H: Reduced parasympathetic control in patients with hypertension at rest and under mental stress. Am. Heart J. 127, 122-128 (1994)

26. Langewitz W, Ruddel H, Schachinger H, Lepper W, Mulder LJ, Veldman JH, van Roon A: Changes in sympathetic and parasympathetic cardiac activation during mental load: an assessment by spectral analysis of heart rate variability. Homeost. Health Dis. 33, 23-33 (1991)

27. Lanza GA, Guido V, Galeazzi MM, Mustilli M, Natali R, Ierardi C, Milici C, Burzotta F, Pasceri V, Tomassini F, Lupi A, Maseri A: Prognostic role of heart rate variability in patients with a recent acute myocardial infarction. Am. J. Cardiol. 82, 1323-1328 (1998)

28. Levy WC, Cerqueira MD, Harp GD, Johannessen K, Abrass IB, Schwartz RS, Stratton JR: Effect of endurance exercise training on heart rate variability at rest in healthy young and older men. Am. J. Cardiol. 82, 1236-1241 (1998)

29. Liao D, Sloan RP, Cascio WE, Folsom AR, Liese AD, Evans GW, Cai J, Sharett AR: Multiple metabolic syndrome is associated with lower heart rate variability. Diab. Care 21, 2116-2122 (1998)

30. Lipsitz LA, Goldberger AL: Loss of 'complexity' and aging. JAMA 267, 1806-1809 (1992)

31. Magari SR, Hauser R, Schwartz J, Williams PL, Smith TJ, Christiani DC: Association of heart rate variability with occupational and environmental exposure to particulate air pollution. Circulation 104, 986-991 (2001)

32. Malik M, Camm AJ: Components of heart rate variability - what they really mean and what we really measure. Am. J. Cardiol. 72, 821-822 (1993)

33. Malik M, Farrell T, Camm AJ: Heart rate variability in relation to prognosis after myocardial infarction: selection of optimal processing techniques. Eur. J. Cardiol. 10, 1060-1074 (1989)

34. Much WAC, Warrien RK, Eschun GM, Girling L, Doiron L, Cheang MS, Lefevre GR: Biologically variable pulsation improves jugular venous oxygen saturation during rewarming. Ann. Thorac. Surg. 69, 491-497 (2000)

35. Mullen TJ, Cohen RJ (1996): RR interval monitoring. In: Noninvasive Electrocardiology. Clinical aspects of Holter monitoring, eds. Moss AJ, Stern S, W. B. Saunders Co. Ltd, London-PhiladelphiaToronto-Sydney-Tokyo, pp. 155-160. 
36. Pagani M, Lucini D, Rimoldi O, Furlan R, Piazza S, Biancardi L, (1995): Effects of physical and mental exercise on heart rate variability. In: Heart Rate Variability, eds. Malik M, Camm AJ, Futura Publishing Co., Inc., Armonk, New York, pp. 250-257.

37. Payne RL, Rick JT: Heart rate as an indicator of stress in surgeons and anaesthetists. J. Psychosom. Res. 30, 411-420 (1986)

38. Pikkujämsä SM, Mäkikallio TH, Sourander LB, Rähilä IJ, Puukka P, Skyttä J, Peng C, Goldberger AL, Huikuri H: Cardiac interbeat interval dynamics from childhood to senescence. Circulation 100, 393-399 (1999)

39. Pruvot E, Thonet G, Vesin JM, van-Melle G, Seidl K, Schmidinger H, Brachmann J, Jung W, Hoffmann E, Tavernier R, Block M, Podczek A, Fromer M: Heart rate dynamics at the onset of ventricular tachyarrhythmias as retrieved from implantable cardioverter defibrillators in patients with coronary artery disease. Circulation 101, 2398-2404 (2000)

40. Ramaekers D, Ector H, Aubert AE, Rubens A, Van de Werf F: Heart rate variability and heart rate in healthy volunteers. Eur. Heart J. 19, 1334-1341 (1998)

41. Redondo M, Del Valle-Inclan F: Decrements in heart rate variability during memory search. Int. J. Psychophysiol. 13, 29-35 (1992)

42. Reinhardt L, Mäkijärvi M, Fetsch T, Martinez-Rubio A, Böcker D, Block M, Borggrefe M, Breithardt G: Reduced beat-to-beat changes of heart rate: an importan risk factor after acute myocardial infarction. Cardiology 87, 104-111 (1996)

43. Rimoldi O, Furlan R, Pagani MR, Piazza S, Guazzi M, Pagani M, Malliani A: Analysis of neural mechanisms accompanying different intensities of dynamic exercise. Chest 101, 226S-230S (1992)

44. Sloan RP, Korten JB, Myers MM: Components of heart rate reactivity during mental arithmetic with and without speaking. Physiol. Behav. 50, 1039-1045 (1991)

45. Task Force of the European Society of Cardiology and the North American Society of Pacing and Electrophysiology: Heart rate variability. Standards of measurement, physiological interpretation, and clinical use. Circulation 93, 1043-1065 (1996)

46. Waller BF, Schlant RC (1994): Anatomy of the heart. In: Hurst's The heart, eds. Schlant RC, Alexander RC, McGraw-Hill Inc., New York, pp. 59-112.

47. Weisz J, Szilagyi N, Lang E, Adam G: The influence of monocular viewing on heart period variability. Int. J. Psychophysiol. 12, 11-18 (1992)

48. Wijbenga JAM, Balk AH, Meij SH, Simoons ML, Malik M: Heart rate variability index in congestive heart failure: relation to clinical variables and prognosis. Eur. Heart J. 19, 1719-1724 (1998)

49. Yeragani VK, Srinivasan K, Vempati S, Pohl R, Balon R: Fractal dimension of heart rate time series: an effective measure of autonomic function. J. Appl. Physiol. 75, 2429-2438 (1993)

50. Ziegler D, Piolot R, Strassburger K, Lambeck H, Dannehl K: Normal ranges and reproducibility of statistical, geometric, frequency domain, and non-linear measures of 24-hour heart rate variability. Horm. Metab. Res. 31, 672-679 (1999) 